\title{
Acting on Harmful Command Hallucinations in Psychotic Disorders
}

\section{An Integrative Approach}

\author{
Frances Shawyer, PhD, *† Andrew Mackinnon, PhD, $\neq$ John Farhall, PhD, $\S$ Eliza Sims, DPsych,* \\ Simone Blaney, PhD, * Priscilla Yardley, DPsych,* Maree Daly, BBSc(Hons), * \\ Paul Mullen, MPhil, DSc, $\dagger$ and David Copolov, MPM, PhD*
}

\begin{abstract}
Although harmful command hallucinations have been linked to violent behavior, few studies have examined factors mediating this relationship. The principal aim of this study was to examine a range of factors potentially associated with acting on harmful command hallucinations using a multivariate approach. The sample comprised 75 participants drawn from community and forensic services. Measures assessing characteristics of the command hallucination and the hallucinator, including forensic risk factors, were administered. Using ordinal logistic regression, we found compliance to be associated with increasing age, viewing the command hallucination as positive, congruent delusions, and reporting low maternal control in childhood. Antipsychotic medication was protective while, contrary to expectations, traditional predictors of violence reduced the odds of compliance with command hallucinations viewed as threatening. The findings suggest that compliance with harmful commands is driven by a complex interaction between beliefs related to the command hallucination and personal characteristics, with risk of compliance increasing with age.
\end{abstract}

Key Words: Schizophrenia, command hallucinations, violence, auditory hallucinations, compliance.

(J Nerv Ment Dis 2008;196: 390-398)

C ommand hallucinations, which are reported by one-half of hallucinating psychiatric patients (Shawyer et al., 2003), can predispose to actions that have harmful conse-

*The Mental Health Research Institute of Victoria, Parkville, Victoria 3052, Australia; †School of Psychology, Psychiatry and Psychological Medicine, Monash University, Victoria, 3800, Australia; $\$$ Centre for Mental Health Research, Australian National University, Canberra, ACT 0200, Australia; $\S$ School of Psychological Science, La Trobe University, Bundoora, Victoria 3086, Australia; and \|Office of the Vice Chancellor, Monash University, Victoria, 3800 Australia.

This research was funded by grant from the National Health and Medical Research Council of Australia (Grant 102846).

Send reprint requests to Frances Shawyer, PhD, Southern Health Adult Psychiatry Research, Training and Evaluation Centre, Dandenong Hospital, PO Box 956, Dandenong, VIC 3175, Australia. E-mail: frances. shawyer@med.monash.edu.au.

Copyright (C) 2008 by Lippincott Williams \& Wilkins

ISSN: 0022-3018/08/19605-0390

DOI: 10.1097/NMD.0b013e318171093b quences. Although acting on command hallucinations is more likely when they are trivial in nature (Chadwick and Birchwood, 1994; Junginger, 1995), compliance with potentially damaging command hallucinations does occur (e.g., Hall et al., 1981; Jones et al., 1992; McNeil et al., 2000; Rogers et al., 2002) and may reflect either the nature of the experience or the individual's pre-existing propensity to violence (Chadwick and Birchwood, 1994).

Factors identified as mediators between command hallucinations and action include coexisting delusions (Mackinnon et al., 2004), particularly delusions that are related to the hallucination (Junginger, 1990), knowing the identity of the voice (Erkwoh et al., 2002; Junginger, 1990, 1995), believing the voices to be real (Erkwoh et al., 2002) and benevolent (1997), number of coping strategies (Mackinnon et al., 2004), not having subjective control over voices (Beck-Sander et al., 1997), and having less frequent auditory hallucinations (Mackinnon et al., 2004). Few studies have specifically investigated factors associated with compliance with potentially harmful command hallucinations. However, the studies by Junginger (1990, 1995) and Beck-Sander et al. (1997) found that the hallucination-related factors associated with compliance were similar for both harmless and harmful commands. In the first published study to focus exclusively on harmful command hallucinations, Fox et al. (2004) found that those who complied were more likely to believe in the voice's power and benevolence. Harm to others was associated with a sense of personal superiority, whereas harm to self was associated with feelings of inferiority.

These findings suggest that compliance with harmful command hallucinations may be a more extreme version of compliance with command hallucinations in general (Braham et al., 2004), with hallucination-related factors providing the key impetus for compliance. However, the results from the study by Fox et al., also point to a potential role for broader factors, such as psychological characteristics of the hallucinator. In general, violent behavior is the result of a complex array of factors (McNeil et al., 2000), yet investigations examining compliance with harmful command hallucinations have included only a small range of predictors primarily related to the hallucination itself. Investigations have also been limited by small sample sizes, especially of noncompli- 


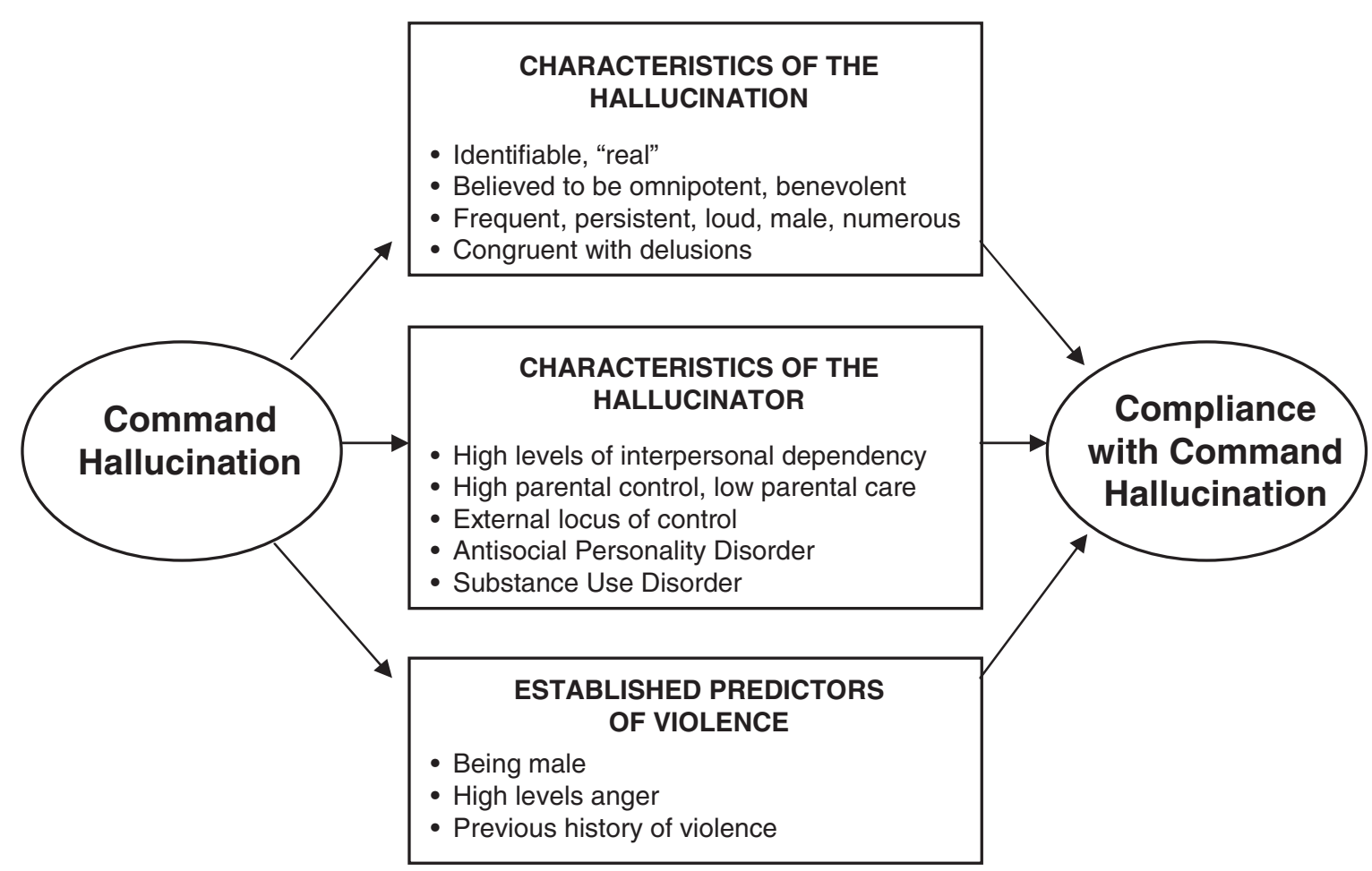

FIGURE 1. Proposed factors mediating compliance with harmful command hallucinations.

ers (Fox et al., 2004; Kasper et al., 1996). These weaknesses are compounded by an absence of multivariate approaches to data analysis. The focus on individual risk factors, or serial univariate analysis of multiple risk factors, gives no information about the independence of sources of risk or the interaction of risk factors.

The aim of the present study was to investigate compliance with harmful command hallucinations using a broad range of predictors within a multivariate framework. Putative risk factors selected for investigation related to both characteristics of the command hallucination and those of the hallucinator, including established predictors of violence (Fig. 1). Because adequate representation of the full range of responses to commands is necessary for the prediction of risk, both forensic and nonforensic mental health populations were accessed to avoid the right-censoring of severity inherent in using only nonforensic patients (Fox et al., 2004).

\section{Hypotheses Related to Characteristics of the Command Hallucination}

\section{Beliefs About Command Hallucinations}

On the basis of the findings reported above, it was hypothesized that compliance would be associated with voices believed to be real (Erkwoh et al., 2002), identifiable (Erkwoh et al., 2002; Junginger, 1990, 1995), and benevolent (Beck-Sander et al., 1997; Birchwood and Chadwick, 1997; Chadwick and Birchwood, 1994, 1995). We also predicted that the belief that the voices are powerful would predict compliance, particularly for voices believed to be threatening or malevolent since these are unlikely to engage intrinsic agreement on the part of the patient (Beck-Sander et al., 1997; Fox et al., 2004).

\section{Phenomenological Characteristics of Command Hallucinations}

Simple "physical" characteristics of the voices are likely to be involved in appraisals of power (Birchwood et al., 2000). Our predictions were that voices that are male, numerous, frequent, persistent, and loud would increase the likelihood of compliance.

\section{Hallucination-Delusion Congruence}

It was hypothesized that patients whose command hallucinations were congruent with their delusions would be more likely to comply since the "logical" basis for action is stronger (Beck-Sander et al., 1997; Bjørkly, 2002; Hersh and Borum, 1998; Junginger, 1990, 1995). Although the logic of the delusion-hallucination congruence is compelling, attempts to investigate this association have been limited by imperfectly operationalized assessments of congruence (Beck-Sander et al., 1997; Junginger, 1990, 1995; Thompson et al., 1992). The present study sought to address this shortcoming through the development of an instrument designed to assess the congruence between command hallucinations and delusions.

\section{Hypotheses Related to Characteristics of the Hallucinator}

\section{Interpersonal Dependence}

Individuals who demonstrate high levels of interpersonal dependence are prone to comply with the expectations and demands of authority figures (Blass, 1991; Bornstein, 1992). Because voices are often considered to be powerful 
and authoritarian, it was predicted that compliance would be associated with high levels of interpersonal dependency.

\section{Reports of Adverse Parenting}

High levels of parental overcontrol and authoritarianism seem to play an important part in determining dependency (Bornstein, 1992). It was therefore hypothesized that patients reporting an affectionless, controlling parental background would be more compliant with command hallucinations than those reporting an affectionate and supportive background.

\section{Locus of Control}

Locus of control is a trait measure tapping the extent to which an individual believes he or she rather than another agent is in control of various domains of life (Levenson, 1973). Patients experiencing auditory hallucinations tend to view them as "not self," or external to their volitional aspect of self (Honig et al., 1998). This, when realized as high levels of external locus of control, was expected to lead to greater compliance.

\section{Comorbidity With Other Psychiatric Disorders}

Although previous research has established an association between personality disorder, substance abuse, psychosis, and violence (Binderman, 1995; Muesser et al., 1997), the mechanisms by which the co-occurrence of these disorders is manifest as violence has not been explored in research to date. It was predicted that participants with a comorbid Antisocial Personality Disorder or Substance Use Disorder would be more likely to be compliant with command hallucinations than those suffering from a psychotic disorder alone.

\section{Hypotheses Related to Established Predictors of Violence}

Male gender having a history of violence and exhibiting high levels of anger are 3 variables that have been almost universally associated with substantial risk of violence in forensic and in-patient psychiatric settings (Monahan and Steadman, 1994). We predicted that these 3 variables would be associated with increased risk of compliance.

\section{METHODS}

\section{Participants}

Adults diagnosed with a DSV-IV psychotic disorder (schizophrenia, schizoaffective disorder, schizophreniform disorder, brief psychotic disorder, psychotic disorder not otherwise specified) or mood disorder with psychotic features, and with a history of harmful command hallucinations, were recruited from forensic and nonforensic mental health services in Melbourne, Australia. Candidates were identified by surveying the case/ward lists of clinicians working within these services on the basis of these criteria. Because command hallucinations are often not reported (Rogers et al., 1990; Zisook et al., 1995), clinicians were encouraged to consider all clients with a history auditory hallucinations. To be eligible to proceed with the study, candidates had to respond positively to a series of screening questions, which aimed to establish a history of harmful command hallucinations. Local research ethics committees approved the study. Written informed consent was obtained from all participants after fully explaining the procedures.

\section{Measures}

Participants were assessed in relation to the maximal level of compliance with the most serious command hallucination experienced since the age of 18 years (the "Index CH"). We evaluated the seriousness of command hallucinations on the basis of its content should it be obeyed. This was judged using a rating scale for dangerousness and by reference to a table of offenses rated according to severity taking into account the maximum penalty set down in legislation in the State of Victoria, Australia. The scale consisted of 4 points in which $1=$ slightly dangerous/serious (e.g., shop steal); 2 = moderately dangerous/serious (e.g., burglary); $3=$ very dangerous/serious (e.g., assault, serious self-harm), and $4=$ extremely dangerous/serious (e.g., murder, suicide). For acts with equivalent ratings of severity, "harm to others" was rated as more serious than harm to self.

\section{Compliance}

Compliance was measured on a 3-point scale; noncompliance, partial compliance (did some but not all of what the voice commanded), and full compliance (participant did all in their power to fulfill the command).

\section{Diagnoses and Illness Severity}

Diagnoses were established using the Structured Clinical Interview for DSM-IV Axis I Disorders (SCID-I) (First et al., 1997b) and illness severity using the Modified Global Assessment of Functioning scale (Hall, 1995). Pharmacotherapy at the time of interview and at the time of the Index $\mathrm{CH}$ was recorded.

\section{Characteristics of the Index Command Hallucination}

All questions addressing characteristics of the Index $\mathrm{CH}$ pertained to beliefs and experience of voices at the time of the Index $\mathrm{CH}$, not at the time of interview. Participants' key beliefs about the voice(s) issuing the Index $\mathrm{CH}$ were assessed using the Beliefs about the Voices QuestionnaireRevised (BAVQ-R-Chadwick et al., 2000). No standardized measures of command hallucinations were available so we developed a structured interview, which included a subset of questions from the Mental Health Unusual Perceptions Scale (Carter et al., 1995) and Psychotic Symptom Rating Scales: Auditory Hallucination subscale (Haddock et al., 1999).

The lack of adequate existing instruments also led us to develop a measure of command hallucination-delusion congruence. All delusions occurring contemporaneously with the Index $\mathrm{CH}$ were assessed as part of the administration of the SCID-I, ensuring the object, action and content of each delusion were recorded clearly. Three-point scales $(0-2)$ were used to evaluate congruence between the command hallucination and each delusion across these 3 categories. The total congruence score was the sum of scores across the 3 
categories for each delusion (range, 0-6). An independent rater, blind to the purpose of the project, performed the ratings. Weighted Kappa between the independent rater and interviewer ratings was 0.85 indicating that the agreement between raters was high.

\section{Characteristics of the Hallucinator}

The Interpersonal Dependency Inventory (Hirschfeld et al., 1977), Parental Bonding Instrument (Parker et al., 1979), and Multidimensional Locus of Control Scale (Levenson, 1973) were administered. Diagnoses for substance use disorders and antisocial personality disorder were established using the SCID-I (First et al., 1997b) and the SCID-II Personality Disorders (First et al., 1997a), respectively. Assessment for substance use disorders was restricted to 12 months before the Index $\mathrm{CH}$.

\section{Established Predictors of Violence}

The tendency to experience anger was measured using the Trait Anger subscale of the State-Trait Anger Expression Inventory-2 (Spielberger, 1999). The history of violence before the Index $\mathrm{CH}$ (since the age of 18) was assessed using the MacArthur Community Violence Instrument (Steadman et al., 1998) but restricted to more serious forms of violence ("Level 1" on the MacArthur Community Violence Instrument) to render it a more focused predictor. (Full details of interview schedules are available from the first author on request).

\section{Statistical Analysis}

Statistical methods for ordered categorical variables were employed (Long, 1997). The first stage of analysis examined individual predictor variables for association with compliance as a screen for the inclusion of variables into multivariate prediction equations. To fully explore the difference between the 2 samples and to maximize statistical power, the community and forensic samples were analyzed together. However, these samples differed substantially in many respects. Accordingly, a variable corresponding to sample source was introduced in all models. Age was also included as an a priori control, since longer time allows greater opportunity for compliance. Multiple ordered logistic regres- sion equations were constructed so that the effects of predictors could be assessed while controlling for other variables.

\section{RESULTS}

\section{Participant Characteristics}

Initial screening resulted in 166 candidates being approached of whom 65 declined and 26 withdrew or were withdrawn from the project after signing consent. The main reasons for withdrawal were: insufficient evidence or poor recall of harmful command hallucinations $(N=12)$, no longer interested $(N=7)$, and mental state instability $(N=5)$. Seventy-five participants ( $56 \mathrm{men}$ ) completed the study, 50 from nonforensic services and 25 from forensic services. The primary diagnosis was schizophrenia (69\%), with most of the remaining participants having an affective psychosis $(28 \%)$.

Participants were receiving a mixture of antipsychotics. The mean daily dose of antipsychotic medication, in chlorpromazine equivalents, was $768 \mathrm{mg}(N=70, S D=433$, range, 83-1800). The mean score on the Modified Global Assessment of Functioning scale was 49 ( $S D=15$, range, 19-90) indicating that, on average, participants experienced some serious symptoms or impairment in functioning. Demographic and psychopathological characteristics for the community and forensic participants are provided in Table 1.

\section{Nature of Index Command Hallucination}

The mean length of time elapsed since the Index $\mathrm{CH}$ to the time of interview was 5.2 years $(S D=6.1)$. The mean duration of auditory hallucinations before the Index $\mathrm{CH}$ was 8.4 years $(S D=9.4)$. Most of the participants $(73 \%)$ had been hospitalized in a psychiatric facility before their Index $\mathrm{CH}$ (mean number of admissions $=3.2, S D=4.2$, range: $0-20)$.

For $48 \%$ of participants, the Index $\mathrm{CH}$ identified involved commands to harm others, $44 \%$ commands to selfharm and, in $8 \%$, to damage property damage or steal. Sixteen $(21 \%)$ participants reported that they did not comply, $23(31 \%)$ complied partially, and $36(48 \%)$ complied fully (Table 2). For those who complied, most (75\%) did so within

\begin{tabular}{lccc}
\hline TABLE 1. Demographic and Psychopathological Characteristics of Community and Forensic Participants \\
\hline & $\begin{array}{c}\text { Community Participants } \\
(\boldsymbol{N}=\mathbf{5 0})\end{array}$ & $\begin{array}{c}\text { Forensic Participants } \\
(\boldsymbol{N}=\mathbf{2 5})\end{array}$ & Statistics \\
\hline Age years [mean $(S D)]$ & $37.1(10.4)$ & $33.6(10.4)$ & $t(73)=1.4 ; \mathrm{NS}$ \\
Sex (male/female) & $33 / 17$ & $23 / 2$ & $\chi^{2}(1)=6.0 ; p=0.02$ \\
Years of education [mean (SD)] & $10.8(2.9)$ & $10.4(2.5)$ & $t(73)=0.7 ; \mathrm{NS}$ \\
Chlorpromazine equivalent dosage in mg & $804.4(462.4)$ & $692.4(364.4)$ & $t(68)=1.0 ; \mathrm{NS}$ \\
$\quad[$ mean $(S D)]$ & $51.8(13.9)$ & $42.5(15.6)$ & $t(68)=1.0 ; p=0.01$ \\
Modified GAF score [mean (SD)] & $3.7(0.8)$ & $3.8(0.5)$ & $t(73)=-0.71 ; p=0.48$ \\
Seriousness of Index CH [mean (SD)] & $3 / 47$ & $13 / 12$ & $\chi^{2}(1)=21.0 ; p=0.00$ \\
Antisocial personality disorder (yes/no) & $17 / 33$ & $15 / 10$ & $\chi^{2}(1)=4.6 ; p=0.03$ \\
Substance use disorder (yes/no) & $17 / 32$ & $17 / 8$ & $\chi^{2}(1)=7.4 ; p=0.007$ \\
History of violence (yes/no) & $2 / 48$ & $20 / 5$ & $\chi^{2}(1)=46.4 ; p=0.00$ \\
Inpatient (yes/no) & &
\end{tabular}


TABLE 2. Number of Participants Showing Each Level of Compliance as a Function of the Object of the Index Command Hallucination (Index $\mathrm{CH}$ )

\begin{tabular}{lcccc}
\hline & \multicolumn{3}{c}{ Object of Index $\mathbf{C H}$} & \\
\cline { 2 - 4 } $\begin{array}{l}\text { Level of } \\
\text { Compliance }\end{array}$ & $\begin{array}{c}\text { Property } \\
\text { Damage/Theft } \boldsymbol{N}\end{array}$ & Self $\boldsymbol{N}$ & Other $\boldsymbol{N}$ & Total $\boldsymbol{N}$ \\
\hline None & 0 & 5 & 11 & 16 \\
Partial & 2 & 10 & 11 & 23 \\
Full & 4 & 18 & 14 & 36 \\
Total & 6 & 33 & 36 & 75 \\
\hline
\end{tabular}

an hour of hearing the Index $\mathrm{CH}$, with a further $19 \%$ complying the same day.

The majority $(83 \%)$ reported a category 4 Index $\mathrm{CH}-$ extremely dangerous/serious (Table 1). Participants' reports of their command hallucinations were checked in 46 cases through a file search, which confirmed $63 \%$ of reports.

\section{Predictors of Compliance}

Many variables and factors that were assessed in the study were not statistically significantly associated with compliance and are not considered below.

\section{Demographic Factors}

Compliance was significantly associated with age (OR per unit increase in age $=1.06, \mathrm{CI}: 1.02-1.11, p=0.009)$ and being a forensic patient $(\mathrm{OR}=2.94, \mathrm{CI}: 1.12-7.71$, $p=0.03)$ but not gender $(\mathrm{OR}=0.83, \mathrm{CI}$ : 0.31-2.23, $p=0.72$ ). Controlling for age, neither age of onset of auditory hallucinations $(\mathrm{OR}=1.04, \mathrm{CI}: 0.97-1.11, p=0.25)$, age at the time of the Index $\mathrm{CH}(\mathrm{OR}=1.00, \mathrm{CI}$ : $0.92-1.08$, $p=0.97)$ nor duration of auditory hallucinations since onset $(\mathrm{OR}=0.97, \mathrm{CI}: 0.92-1.03, p=0.28)$ were significantly related to compliance.

\section{Characteristics of Command Hallucinations}

Compliance did not vary as a function of the object of the command $\left(\chi^{2}=4.7, d f=4\right.$, exact $\left.p=0.33\right)$ nor did "self-harm" versus "harm others" commands predict compliance $(\mathrm{OR}=2.05, \mathrm{CI}: 0.83-5.04, p=0.12)$. Table 3 shows the results of the regression analyses for the characteristics of command hallucinations adjusted for age and sample source.

Overall, participants reported much higher levels of resistance to their Index $\mathrm{CH}$ than engagement (mean BAVQ-R resistance $=17.1, S D=7.0$; mean BAVQ-R engagement $=7.5, S D=5.6$ ). Participants appraising their experience of the Index $\mathrm{CH}$ as irrelevant, positive (of benefit to them) or challenging (of benefit, but also stressful) rather than as a threat were more likely to comply. In fact, only 1 of the 27 participants who characterized their voices in 1 of the first 3 categories did not comply compared with 15 of the 46 who characterized their voices as a threat. All participants who described the experience of the Index command as positive $(N=13)$ complied either partially $(N=3)$ or fully $(N=10)$. There was a trend for participants with low scores on the sum of negative tone items (see Copolov et al., 2004) to be more likely to comply.
Although power, as assessed by the BAVQ-R omnipotence scale, did not predict compliance, a number of phenomenological characteristics of hallucinations did so. There was no simple effect of sex of the voice but participants whose Index $\mathrm{CH}$ came from voice(s) that were clearly not female were more likely to comply. The influence of frequency was complex with compliance being highest in situations where either the commands were heard only on one occasion or where it was frequently repeated. Compliance was least where the command hallucination was reported to occur occasionally (Table 3). Absence of other harmful command hallucinations around the time of the Index $\mathrm{CH}$ also increased likelihood of compliance: 12 (75\%) of the noncompliers heard other dangerous command hallucinations compared with $9(39 \%)$ partial compliers and 13 (36\%) full compliers. Total hallucination-delusion congruence score predicted compliance.

\section{Characteristics of the Hallucinator and Established Predictors of Violence}

Only the Maternal Overprotection scale had a significant relationship with compliance, where low control predicted compliance (OR per unit decrease in PBI maternal overprotection $=1.08$, CI 1.02-1.15, $p=0.01$, controlling for age and source).

\section{Antipsychotic Medication}

At the time of the Index $\mathrm{CH}, 39$ (53\%) participants were being treated with antipsychotic medication (mean daily dose chlorpromazine equivalents $852 \mathrm{mg}$, range, 40-1800). Those who were not taking antipsychotic medication at the time of the Index $\mathrm{CH}$ were more likely to comply $(\mathrm{OR}=5.37, \mathrm{CI}: 1.89-15.20, p=0.002$, controlling for age and sample source).

\section{Multiple Logistic Regression Analyses}

Although the sample size restricted the construction of complex models, we sought to determine the incremental value of combining predictors of compliance. Variables were selected according to their potential clinical utility, conditional on having demonstrated a significant univariate association with compliance. In some cases, the combination of modest sample size and the distribution of the predictors, individually and in combination, meant that stable and reliable results could not be obtained in models with multiple predictors. In these cases, analyses were simplified by reducing the number of variables included or by recoding variables using broader classifications. Clinical characteristics of the hallucinations that could be readily assessed were entered first into the regression equation followed by features of the hallucinations that would normally require more extensive investigation, such as hallucination-delusion congruence. These models were run by forcing age and participant source in the equation and applying forward and backward selection methods, used as heuristics, to the other variables. To limit the number of variables for analysis, frequency of Index $\mathrm{CH}$ around the time of the Index $\mathrm{CH}$ was included in the regression analysis in preference to frequency of Index $\mathrm{CH}$ on the day for reasons of clinical utility. "Threat versus positive" 
TABLE 3. Ordinal Regression Results for Characteristics of Command Hallucinations as Predictors of Compliance, Adjusted for Age and Sample Source

\begin{tabular}{|c|c|c|c|}
\hline Characteristic of Command Hallucinations & Odds Ratio & 95\% Confidence Interval & $p$ \\
\hline \multicolumn{4}{|l|}{ Beliefs about voices } \\
\hline Identity & 0.94 & $0.38-2.34$ & 0.890 \\
\hline \multicolumn{4}{|l|}{ BAVQ-R } \\
\hline Resistance $^{\mathrm{a}}$ & 1.09 & $1.02-1.18$ & 0.018 \\
\hline Engagement & 1.09 & $1.00-1.19$ & 0.048 \\
\hline Benevolence & 1.02 & $0.93-1.12$ & 0.717 \\
\hline Malevolence $^{\mathrm{a}}$ & 1.09 & $0.99-1.21$ & 0.075 \\
\hline Omnipotence & 1.07 & $0.95-1.21$ & 0.284 \\
\hline \multicolumn{4}{|l|}{ Appraisal } \\
\hline Threat vs. positive/irrelevant/challenging & 3.46 & $1.24-9.70$ & 0.018 \\
\hline Threat vs. positive & 7.39 & $1.52-35.95$ & 0.013 \\
\hline Negative tone $\mathrm{a}^{\mathrm{a}}$ & 1.28 & $0.99-1.63$ & 0.055 \\
\hline Insight & 0.93 & $0.60-1.43$ & 0.743 \\
\hline \multicolumn{4}{|l|}{ Phenomenological characteristics } \\
\hline Gender: voices not identifiably female & 3.44 & $1.32-8.98$ & 0.012 \\
\hline \multicolumn{4}{|l|}{ Number } \\
\hline Number of voices issuing Index $\mathrm{CH}^{\mathrm{a}}$ & 0.82 & $0.50-1.34$ & 0.426 \\
\hline Hearing fewer voices around time & 1.92 & $1.22-3.03$ & 0.005 \\
\hline \multicolumn{4}{|l|}{ Frequency } \\
\hline $\begin{array}{l}\text { Index } \mathrm{CH} \text { once/regularly (vs. occasionally } \\
\text { around time) }\end{array}$ & 6.13 & $1.28-29.37$ & 0.023 \\
\hline $\begin{array}{l}\text { Index } \mathrm{CH} \text { once/very frequently (vs. several } \\
\text { times on day) }\end{array}$ & 4.64 & $1.61-13.34$ & 0.004 \\
\hline Absence of other harmful $\mathrm{CHs}$ around time & 3.21 & $1.22-8.45$ & 0.018 \\
\hline Persistence: time over which Index $\mathrm{CH}$ heard ${ }^{\mathrm{b}}$ & 0.89 & $0.76-1.05$ & 0.159 \\
\hline Loudness & 1.14 & $0.71-1.82$ & 0.589 \\
\hline \multicolumn{4}{|l|}{ Location } \\
\hline Outside vs. inside \& inside/outside & 1.73 & $0.64-4.71$ & 0.283 \\
\hline Inside vs. outside \& inside/outside & 1.29 & $0.49-3.41$ & 0.604 \\
\hline \multicolumn{4}{|l|}{ Hallucination-delusion congruence } \\
\hline Object congruence & 1.73 & $0.85-3.54$ & 0.131 \\
\hline Content congruence & 2.22 & $0.87-5.69$ & 0.096 \\
\hline Action congruence & 2.19 & $1.00-4.82$ & 0.051 \\
\hline Total congruence & 1.66 & $1.14-2.41$ & 0.008 \\
\hline
\end{tabular}

was included in preference toöthreat versus irrelevant/positive/challenging," because of its stronger univariate association with compliance.

Both forward and backward selection produced models containing age, source and frequency of the Index $\mathrm{CH}$ around the time of the command. The forward method added gender of voice. Adding this variable had little effect on the values of the other parameters, so it was retained in the model. The complex clinical variables, "appraisal" (threat versus positive) andöcongruence with delusions, " were entered in separate equations with the significant "simple" variables retained. Approached in this way, both variables were significant. Gender, source, and frequency did not consistently reach significance in these models and so were eliminated from further analyses.

Maternal overprotection was then added to the equation containing the surviving factors of age, "appraisal" and "con- gruence with delusions." This produced the final model for primary analyses in which all 4 variables were significant (age: $\quad \mathrm{OR}=1.09, \mathrm{CI}: 1.02-1.17, p=0.011$; appraisal: $\mathrm{OR}=16.17, \mathrm{CI}: 2.43-107.45, p=0.004$; congruence: OR per unit increase $=1.74$, CI: $1.12-2.72, p=014$; maternal overprotection: OR per unit decrease $=1.10$, CI: $1.02-1.19$, $p=0.018$ ).

The effect of antipsychotic medication was also examined in an additional, exploratory analysis. Antipsychotic medication was significant when added to the above 4 factors $(\mathrm{OR}=9.09$; CI: 2.10-39.29, $p=0.003)$ but maternal overprotection was not.

\section{Subsidiary Analyses}

We were interested in examining further the predictive value of perceived power of the voice in the context of threatening voices. Beliefs about power are of most relevance 
in this circumstance since threatening voices are unlikely to engage intrinsic agreement on the part of the patient (BeckSander et al., 1997; Fox et al., 2004). An interaction term was added to a model containing the BAVQ-R omnipotence scale and whether or not the voice was threatening. As predicted, the interaction was significant $\left(\chi^{2}=10.70, d f=1, p=0.001\right)$. Responses for participants who described their voices threatening $(N=46)$ were therefore analyzed separately. For this subgroup, those who had higher scores on the omnipotence scale were more likely to comply with their command hallucinations than participants with lower scores, controlling for age and source (OR, unit change in BAVQ-R Omnipotence $=1.23$, CI: $1.02-0.56, p=0.03)$.

Each of the other predictors was also re-examined within this subgroup, controlling for age and source. Overall, results replicated the findings for the sample as a whole and increased the ORs. In addition, having no history of violence increased the odds of compliance for the threat group $(\mathrm{OR}=3.99, \mathrm{CI}: 1.07-14.88, p=0.04$, controlling for age and source). Only 3 of the $18(17 \%)$ total compliers had a history of violence compared with 7 (40\%) noncompliers and $6(54 \%)$ partial compliers. The odds were increased further by the inclusion of BAVQ-R omnipotence in the equation $(\mathrm{OR}=7.07, \mathrm{CI}: 1.68-29.79, p=0.008)$. Given this unexpected inverse relationship between history of violence and compliance, the nexus between BAVQ-R omnipotence, anger and compliance was similarly explored controlling for age and source. Omnipotence (OR, unit increase BAVQ-R Omnipotence scale $=1.28, \mathrm{CI}: 1.04-1.57, p=0.02$ ) and trait anger (OR, unit decrease in STAXI Trait Anger $=1.17, \mathrm{CI}$ : $1.00-1.35, p=0.04)$ significantly predicted compliance.

The unexpected direction of these findings alongside those related to maternal overprotection prompted us to examine, for the sample as a whole, the interrelationship between maternal overprotection and anger and having history of violence. Trait anger and maternal overprotection were unrelated across all subscales. However, there was a trend for those with a history of violence to report higher levels of maternal control than those without a history of violence $(t=-1.98, d f=70, p=0.051)$.

It was not possible to undertake regression analysis in the subgroup who viewed their experience of the Index $\mathrm{CH}$ as nonthreatening because of small numbers and the almost complete absence of noncompliers. However, in an effort to understand this group's high levels of compliance, we examined differences between compliers with $(N=31)$ and without $(N=26)$ threatening voices. Results showed that compared with participants with threatening voices, those with positive voices had higher levels of trait anger $(t=2.10$, $d f=41, p=0.04)$, whereas those with positive/irrelevant/ challenging voices were more likely to report a history of violence $(32 \%$ vs. $68 \%)\left(\chi^{2}=7.08, d f=1, p=0.01\right)$.

\section{DISCUSSION}

Most of the participants in this study viewed their harmful command hallucinations as threatening and reported high levels of resistance, though many still acted on the voices to some extent. Although prevalence rates for at least partial compliance with command hallucinations vary markedly depending upon the sample and methodology (Shawyer et al., 2003), our compliance rate of $79 \%$ is toward the high end of the range previously reported (range, $0 \%-92 \%$ ). This is probably not surprising given that we were seeking to identify episodes of maximal compliance over the adult lifetime.

What is perhaps more surprising is that although the command hallucinations were invariably advocating harm, nearly $40 \%$ of participants regarded the Index $\mathrm{CH}$ as neutral, positive, or at most, challenging. Furthermore, the positive appraisal of the Index $\mathrm{CH}$ emerged as a key predictor of compliance. Our findings clearly run counter to a simple characterization of compliance with command hallucinations as automatic submission to threats or coercion. Developmental and forensic risk factors also affected the chances of compliance, particularly for threatening voices; however, the direction of effect was opposite to that which was expected. While viewing the voices as a threat was generally protective, this effect was likely to be overridden if the voice was perceived to be very powerful or if the voice hearer had low levels of anger and aggression. We also found that phenomenological factors predicted compliance in the univariate analysis. However, the significant effects of these "simple" clinical factors declined when the more complex beliefs were included in the analysis.

There is evidence to suggest that violence is much more likely to be motivated by delusions than hallucinations (Swanson et al., 1996; Taylor, 1985; Taylor et al., 1998), that individuals who comply with command hallucinations are more likely than noncompliers to be assessed as having delusions (Mackinnon et al., 2004), and that any association between command hallucinations and violence is likely to operate through aspects of delusional beliefs (Junginger, 1990, 1995; Junginger and McGuire, 2004). The current study extends these findings to show that the odds of compliance with harmful command hallucinations are increased for individuals with congruent delusions. Although such a relationship has been suggested by others, this is the first clear and quantified demonstration of it using a direct and independently applied measure of congruence.

Lower levels of maternal control predicted compliance, regardless of levels of care. High levels of maternal control may operate to protect against compliance with harmful command hallucinations by fostering a reciprocal defiant attitude and resistant behavior. In support of this interpretation, high levels of anger and having a history of violence was protective against threatening, powerful voices and there was a trend for those with a history of violence to have higher levels of maternal control than those without a history of violence.

Although speculative, the results of the subsidiary analysis suggest that the negative relationship between anger and history of violence and compliance may not hold for those with nonthreatening voices and may even be in the reverse direction. Future studies distinguishing predictors of compliance for nonthreatening and threatening voices could be of significant clinical utility and may help explain the disso- 
nance between the harmful content of commands and their positive appraisal.

The overall pattern of findings is consistent with observations made by Junginger (1990) in demonstrating that those who comply have a more severe psychotic disturbance. The finding that antipsychotic medication was protective also supports this interpretation. However, antipsychotic medication, taken at the time of compliance, was far from being fully effective. Thirty-six percent of those who fully complied (46\% of partial/full compliers) were taking antipsychotic medication at the time. In addition, as has been reported by others (Jones et al., 1992; Karp et al., 1991; Shore et al., 1978), hospitalization was also not an absolute barrier to compliance. Four of the 6 participants who were in hospital at the time of their Index CH complied, 2 fully. Given that $71 \%$ of full compliers had been previously hospitalized, the risk of harm from command hallucinations does not seem to be greatest at the inception of illness.

Although prospective studies of compliance are not practical given the expense and difficulties involved [such as the obligation of clinicians to take preventive steps when patients report dangerous command hallucinations (Bjørkly, 2002; Junginger, 1995; McNeil et al., 2000; Rogers et al., 2002)], retrospective studies such as this need to be interpreted cautiously. Because of our interest in the link between command hallucinations and behavior, which is serious at the criminal level, we could not address recall bias by restricting the Index $\mathrm{CH}$ to a recent occurrence. We were able to substantiate the existence of the Index $\mathrm{CH}$ through a file search for $63 \%$ of participants. Although this level of substantiation is consistent with previous studies (Rogers et al., 1990; Zisook et al., 1995), it does not fully address memory bias in relation to the details of the event. To a large extent then, this work rests on the assumption that, by virtue of its seriousness, the experience of the Index $\mathrm{CH}$ would retain prominence in memory.

Retrospective reports, however, are naturally subject to memory distortions (Mott et al., 1965). For example, for participants who complied, the ensuing process of effort after meaning (Bartlett, 1932) may have strengthened certain beliefs the patient had about the event and weakened others. Although the results show that participants with threatening voices were more likely to comply with powerful voices, it may be that they rated the voices they complied with as more powerful (Fox et al., 2004). It might be noted, however, that at least some of the predictors would seem to be less susceptible to effort after meaning. These include anger, history of violence, and maternal overprotection since these are general measures rather than linked to the Index $\mathrm{CH}$. Delusion congruence is also less susceptible to effort after meaning since the link between delusions and the Index $\mathrm{CH}$ was made by an independent rater. A final limitation to note is the use of a sample drawn from services in a single geographic region (Melbourne, Australia). As with any such research, care should be exercised in generalizing our findings to individuals with harmful command hallucinations in other populations.
Balanced against its limitations are a number of strengths. Taking a "close-in" approach (Bell, 1992), we have focused on a specific experience of a harmful command hallucination rather than the general experience (cf., Fox et al., 2004) and completed a comprehensive examination of a range of predictors using multivariate statistical techniques. Previous studies have generally provided a simple breakdown of the measures collected. This information does not convey the likelihood of individuals having particular characteristics acting on a command hallucination. The focus on individual risk factors also gives no information about the interaction of risk factors. Although still modest, of the number of participants in this study with harmful command hallucinations is the largest yet published. The sample also includes good representation from the forensic population which redresses the right-censoring of severity inherent in using only community-based participants.

\section{CONCLUSIONS}

Recent reviews (Bjørkly, 2002; Braham et al., 2004) concluded that identifying mediating factors is critical to understanding and predicting compliance with harmful command hallucinations. This study sought to examine a broad range of predictors derived from the literature and clinical experience. Our findings indicate that beliefs related to the command hallucination were particularly important for compliance. These include viewing the command hallucination as being of benefit to the individual, and having delusions that were congruent with the action being prescribed. Furthermore, when participants viewed their command hallucination as a threat, the belief that the voice was powerful predicted compliance, overriding the natural tendency to resist such a threat. Critically, beliefs about the command were more important for prediction than the actual phenomenology of the experience. Beliefs interacted with developmental and forensic characteristics in ways which were not expected given the literature. For example, reporting a history of high maternal control was generally protective, as was having a history of violence and high levels of anger for those who viewed their command hallucination as threatening and powerful. There seems to be different pathways to compliance as a function of whether the command hallucination is viewed as positive or a threat.

Although limited by the modest sample recruited, the current study demonstrates the importance of taking a broad, integrative approach to the assessment of risk of acting on hallucinations. It is clear that multiple factors are involved and that these interact in ways that are sometimes complex. Future research distinguishing the multiple pathways to action and resistance would be of significant clinical utility.

\section{REFERENCES}

Bartlett FC (1932) Remembering. A Study in Experimental and Social Psychology. Cambridge: Cambridge University press.

Beck-Sander A, Birchwood M, Chadwick P (1997) Acting on command hallucinations: A cognitive approach. Br J Clin Psychol. 36:139-148.

Bell RQ (1992) Multiple-risk cohorts and segmenting risk as solutions to the problem of false positives in risk for major psychoses. Psychiatry. 55: $370-381$. 
Binderman RM (1995) Identifying violence risk factors. In L VandeCreek, S Knapp, TL Jackson (Eds), Innovations in Clinical Practice: A Source Book (Vol 14). Sarasota: Professional Resource Press.

Birchwood M, Chadwick P (1997) The omnipotence of voices: Testing the validity of a cognitive model. Psychol Med. 27:1345-1353.

Birchwood M, Meaden A, Trower P, Gilbert P, Plaistow J (2000) The power and omnipotence of voices: Subordination and entrapment by voices and significant others. Psychol Med. 30:337-344.

Bjørkly S (2002) Psychotic symptoms and violence toward others-a literature review of some preliminary findings Part 2. Hallucinations. Aggress Violent Beh. 7:605-615.

Blass T (1991) Understanding behaviour in the milgram obedience experiment: The role of personality, situations and their interactions. $J$ Pers Soc Psychol. 60:398-413.

Bornstein RF (1992) The dependent personality: Developmental, social and clinical perspectives. Psychol Bull. 112:3-23.

Braham LG, Trower P, Birchwood M (2004) Acting on command hallucinations and dangerous behavior: A critique of the major findings in the last decade. Clin Psychol Rev. 24:513-528.

Carter DM, Mackinnon A, Howard S, Zeegers T, Copolov DL (1995) The development and reliability of the Mental Health Research Institute Unusual Perceptions Schedule (MUPS): An instrument to record auditory hallucinatory experience. Schizophr Res. 16:157-165.

Chadwick P, Birchwood M (1994) The omnipotence of voices: A cognitive approach to auditory hallucinations. Br J Psychiatry. 164:190-201.

Chadwick P, Birchwood M (1995) The omnipotence of voices II: The Beliefs About Voices Questionnaire (BAVQ). Br J Psychiatry. 166:773-776.

Chadwick P, Lees S, Birchwood M (2000) The revised beliefs about voices questionnaire (BAVQ-R). Br J Psychiatry. 177:229-232.

Copolov D, Mackinnon A, Trauer T (2004) Correlates of the affective impact of auditory hallucinations in psychotic disorders. Schizophr Bull. 30:163-171.

Erkwoh R, Willmes K, Eming-Erdmann A, Kunert HJ (2002) Command hallucinations: Who obeys and who resists them? Psychopathology. 35: 272-279.

First MB, Gibbon M, Spitzer RL, Williams JBW, Benjamin LS (1997a) Structured Clinical Interview for DSM-IV Axis II Personality Disorders (SCID-II). Washington (DC): American Psychiatric Press.

First MB, Spitzer RL, Gibbon M, Williams JBW (1997b) Structured Clinical Interview for DSM-IV Axis I Disorders. Washington (DC): American Psychiatric Press.

Fox JRE, Gray NS, Lewis H (2004) Factors determining compliance with command hallucinations with violent content: The role of social rank, perceived power of the voice and voice malevolence. J Forensic Psychiatr Psychol. 15:511-531.

Haddock G, McCarron J, Tarrier N, Faragher EB (1999) Scales to measure dimensions of hallucinations and delusions: The psychotic rating scale (PSYRATS). Psychol Med. 29:879-889.

Hall DC, Lawson BZ, Wilson LG (1981) Command hallucinations and self-amputation of the penis and hand during a first psychotic break. $J$ Clin Psychiatry. 42:322-324.

Hall RCW (1995) Global assessment of functioning: A modified scale. Psychosomatics. 36:267-275.

Hersh K, Borum R (1998) Command hallucinations, compliance and risk assessment. J Am Acad Psychiatry Law. 26:353-359.

Hirschfeld RMA, Klerman GL, Gough HG, Barrett J, Korchin SJ, Chodoff P (1977) A measure of interpersonal dependency. J Pers Assess. 41:610-618.

Honig A, Romme MAJ, Ensink BJ, Escher SDMA, Pennings MHA, Devries MW (1998) Auditory hallucinations: A comparison between patients and nonpatients. J Nerv Ment Dis. 186:646-651.

Humphreys MS, Johnstone EC, MacMillan JF, Taylor PJ (1992) Dangerous behaviour preceding first admissions for schizophrenia. $\mathrm{Br} J$ Psychiatry. 161:501-505.
Jones G, Huckle P, Tanaghow A (1992) Command hallucinations, schizophrenia and sexual assaults. Ir J Psychol Med. 9:47-49.

Junginger J (1990) Predicting compliance with command hallucinations. Am J Psychiatry. 147:245-247.

Junginger J (1995) Command hallucinations and the prediction of dangerousness. Psychiatr Serv. 46:911-913.

Junginger J, McGuire P (2004) Psychotic motivation and the paradox of current research on serious mental illness and rates of violence. Schizophr Bull. 30:21-30.

Karp JG, Whitman L, Convit A (1991) Intentional ingestion of foreign objects by male prison inmates. Hosp Community Psychiatry. 42:533-535.

Kasper ME, Rogers R, Adams PA (1996) Dangerousness and command hallucinations: An investigation of psychotic symptoms. Bull Am Acad Psychiatry Law. 24:219-224.

Levenson H (1973) Multidimensional locus of control in psychiatric patients. $J$ Consult Clin Psychol. 41:397-404.

Long JS (1997) Regression Models for Categorical and Limited Dependent Variables. Thousand Oaks (CA): Sage.

Mackinnon A, Copolov DL, Trauer T (2004) Factors associated with compliance and resistance to command hallucinations. J Nerv Ment Dis. 192:357-362.

McNeil DE, Eisner JP, Binder RL (2000) The relationship between command hallucinations and violence. Psychiatr Serv. 51:1288-1292.

Monahan J, Steadman HJ (Eds) (1994) Violence and Mental Disorder: Developments in Risk Assessment. Chicago: University of Chicago Press.

Mott RH, Small IF, Anderson JM (1965) Comparative study of hallucinations. Arch Gen Psychiatry. 12:595-601.

Muesser KT, Drake RE, Ackerson TH, Alterman AI, Miles KM, Noordsy DL (1997) Antisocial personality disorder, conduct disorder and substance abuse in schizophrenia. J Abnorm Psychol. 106:473-477.

Parker G, Tupling H, Brown LB (1979) A parental bonding instrument. Br J Med Psychol. 52:1-10.

Rogers P, Watt A, Gray NS, MacCulloch M, Gournay K (2002) Content of command hallucinations predicts self-harm but not violence in a medium secure unit. J Forensic Psychiatry. 13:251-262.

Rogers R, Gillis R, Turner E, Frise-Smith T (1990) The clinical presentation of command hallucinations in a forensic population. Am J Psychiatry. 147:1304-1307.

Shawyer F, Mackinnon A, Farhall J, Trauer T, Copolov D (2003) Command hallucinations and violence: Implications for detention and treatment. Psychiatr Psychol Law. 10:97-107.

Shore D, Anderson DJ, Cutler NR (1978) Prediction of self-mutilation in hospitalized schizophrenics. Am J Psychiatry. 135:1406-1407.

Spielberger CD (1999) State-Trait Anger Expression Inventory-2: Professional Manual. Odessa (FL): Psychological Assessment Resources, Inc.

Steadman HJ, Mulvey EP, Monohan J, Robbins PC, Appelbaum PS, Grisso T, Roth LH, Silver E (1998) Violence by people discharged from acute psychiatric inpatient facilities and by others in the same neighborhoods. Arch Gen Psychiatry. 55:393-401.

Swanson JW, Borum R, Swartz M, Monahan J (1996) Psychotic symptoms and disorders and risk of violent behaviour in the community. Crim Behav Ment Health. 6:309-329.

Taylor PJ (1985) Motives for offending among violent and psychotic men. Br J Psychiatry. 147:491-498.

Taylor PJ, Leese M, Williams D, Butwell M, Daly R, Larkin E (1998) Mental disorder and violence. A special (high security) hospital study. Br J Psychiatry. 172:218-226.

Thompson JS, Stuart GL, Holden CE (1992) Command hallucinations and legal insanity. Forensic Rep. 5:29-43.

Zisook S, Byrd D, Kuck J, Jeste DV (1995) Command hallucinations in outpatients with schizophrenia. J Clin Psychiatry. 56:462-465. 